\title{
Analysis of Ultrasonic Transducers with Fractal
}

\author{
Architecture
}

\author{
Leigh-Ann Orr $^{\dagger}$, Anthony J. Mulholland ${ }^{\dagger *}$, Richard L. O’Leary ${ }^{\ddagger}$ \\ and Gordon Hayward ${ }^{\ddagger}$ \\ $\dagger$ Department of Mathematics, University of Strathclyde, \\ Glasgow, UK G1 1XH \\ $\ddagger$ Department of Electronic and Electrical Engineering, \\ University of Strathclyde, Glasgow, UK G1 1XW
}

November 26, 2007

\begin{abstract}
Ultrasonic transducers composed of a periodic piezoelectric composite are generally accepted as the design of choice in many applications. Their architecture is normally very regular and this is due to manufacturing constraints rather than performance optimisation. Many of these manufacturing restrictions no longer hold due to new production methods such as computer controlled, laser cutting, and so there is now freedom to investigate new types of geometry. In this paper, the plane wave expansion model is utilised to investigate the behaviour of a transducer with a self-similar architecture. The Cantor set is utilised to design a 2-2 configuration, and a 1-3 configuration is investigated with a Sierpinski Carpet geometry.
\end{abstract}

*Department of Mathematics, University of Strathclyde, Livingstone Tower, 26 Richmond Street, Glasgow G1 1XH, U.K., Tel: ++44 (0)141 548 2971, Fax: ++44 (0)141 548 3345, email: ajm@maths.strath.ac.uk 
Ideally a single longitudinal mode in the thickness direction will drive the transducer in a piston like fashion. In this paper it was found that by increasing the fractal generation level, the bandwidth surrounding the main thickness mode will increase, but there will be a corresponding reduction in the amplitude of the electrical conductance. It is also shown that a shift in the frequency of operation of the device can be achieved by altering the spatial periodicity of the electrical excitation.

Keywords: FRACTAL, ULTRASONIC TRANSDUCER, PLANE WAVE EXPANSION

\section{Introduction}

Ultrasonic transducers composed of a periodic piezoelectric composite are generally accepted as the design of choice in many biomedical [1], sonar [2] and nondestructive testing applications [3]. This is due to the constituent materials combining to realise better operational characteristics, coupled with the availability of new materials $[4,5]$. The most frequently used designs are manufactured by dicing the ceramic into a series of pillars and then filling the void with a passive polymer phase [6]. The 1-3 design has connectivity in only one direction for the ceramic phase but in all three directions for the polymer phase (see Figure1(a)). For the 2-2 design, the ceramic is cut longitudinally in one direction so that there is connectivity in two directions for both the ceramic and polymer phases (see Figure1(b)). Ideally a single longitudinal mode in the thickness direction will drive the transducer in a piston like fashion. Other modes, propagating in other directions, can interfere with this behaviour and hence it is of interest to theoretically predict the design criteria, material parameters, etc. that will ensure a large frequency band gap between the desired thickness mode and these other waves. Note however that the standard classification of the modes is problematic in this setting as the supporting medium is heterogeneous, anisotropic, lossy and piezoelectric. As such the descriptions of the waves in terms of their symmetry, or as Lamb, Rayleigh, bulk waves etc. are only 
pseudo-descriptions and the actual behaviour is more complex.

It is also desirable to maximise the range of frequencies over which the device can operate; to increase the operational bandwidth. This can aid the generation of coded excitations that are used to improve the spatial resolution and signal to noise ratios $[7,8,9]$. These coded signals are very complex and often require the device to be able to produce vibrations over a wide range of frequencies. Hence it is of interest to investigate new transducer designs that can realise improved bandwidths. The use of second harmonic imaging with ultrasound contrast agents (UCA) also requires a broadband device to generate the chirp excitation [10]. As transducers operate around a fixed frequency, one transducer is used to transmit the excitation wave (at the first harmonic frequency) and a separate transducer, with a centre frequency at the second harmonic of the UCA is used to receive the reflected signal. This is a typical situation in ultrasound applications, in that set of devices, each one operating at a different frequency, is required to deal with each new application. It would therefore be desirable to be able to switch the centre frequency of a device so that it could be used in a wider variety of settings.

The traditional designs used in ultrasound transducers are very regular and have arisen due to manufacturing constraints rather than performance optimisation. Many of these restrictions no longer hold due to new manufacturing processes such as computer controlled, laser cutting machines, and so there is now freedom to investigate new types of geometry. Devices with irregular and self-similar constructions may prove beneficial however their use for ultrasonic transducer and array design has not been investigated before. In this paper, the plane wave expansion model (PWE) $[11,12]$ is utilised to investigate the behaviour of a transducer with a fractal architecture. Two designs which exhibit selfsimilarity are investigated for their suitability as new transducer array designs. Firstly the Cantor set, is utilised to design a 2-2 configuration, where each new fractal generation level will introduce additional ceramic pillars into the transducer (see Figure 3(b)). Secondly a 1-3 configuration will be investigated with a Sierpinski Carpet geometry (see Figure 3(a)). The results show an increase in the transmission bandwidth and an ability to switch the frequency of operation of the device. This work also contributes to the 


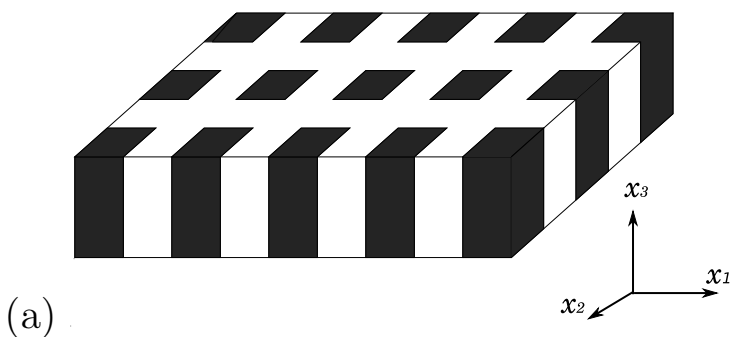

(b)

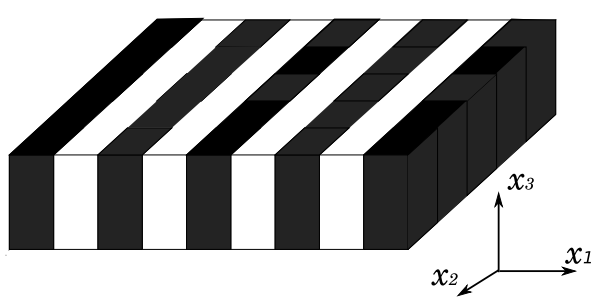

Figure 1: Schematic of a periodic composite transducer where the piezoelectric ceramic pillars are in black and the polymer filler is in white, (a) 1-3 topology, (b) 2-2 topology growing body of literature on wave propagation in fractal media $[13,14,15,16,17,18]$.

\section{Formulation of the method}

In the next section the partial differential equations that describe the physical model of the transducer are shown, along with their associated boundary conditions. A simplified model of the transducer is presented that does not include a matching or backing layer, nor any electrical or mechanical loads. The geometry of the transducer is described in terms of a Fourier series and this, coupled with the Plane Wave Expansion (PWE) method, is used to find a solution to these equations.

\subsection{The Physical Model and Boundary Conditions}

The piezoelectric constitutive equations, together with Newton's second law and Gauss's law for dielectric media are [19]

$$
\begin{aligned}
T_{i j} & =c_{i j k l} u_{k, l}+e_{l i j} \phi_{, l} \\
D_{i} & =e_{i k l} u_{k, l}-\epsilon_{i l} \phi_{, l} \\
\rho \frac{\partial^{2} u_{j}}{\partial t^{2}} & =T_{i j, i} \\
D_{i, i} & =0
\end{aligned}
$$


where $c_{i j k l}$ is the elastic modulus tensor, $e_{l i j}$ is the piezoelectric stress tensor, and $\epsilon_{i l}$ is the permittivity tensor. Equations (1) to (4) constitute 16 equations in the 16 unknowns which are the stresses $T_{i j}$, the displacements $u_{k}$, the electric potential $\phi$ and the electrical displacements $D_{i}$. The method is sufficiently general to cope with a wide range of boundary conditions but for simplicity the mechanical boundary conditions of a stress free plate are considered. For the electrical boundary conditions the electrical

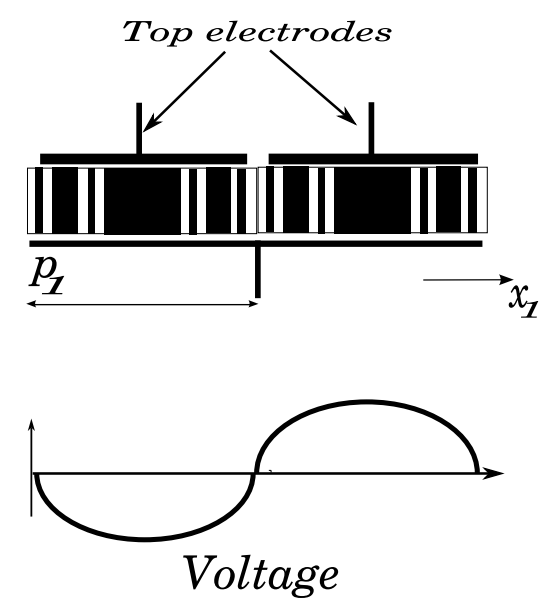

Figure 2: The top electrode spacing and an example applied voltage when $\gamma_{1}=$ $k_{1} p_{1} /(2 \pi)=1 / 2$.

potentials at the top and bottom of the transducer are prescribed, along with continuity of the electrical potential at the front interface. The lower surface is a monolithic plate with zero electrical potential. The upper plate has a set of electrodes which follow the periodic spatial pattern of the ceramic pillars. The top surface electrical potential is therefore described by

$$
\phi\left(x_{1}, x_{2}, t\right)=V_{0} e^{\jmath\left(\omega t-\gamma_{1} x_{1}-\gamma_{2} x_{2}\right)}
$$

where $\gamma_{i}=k_{i} p_{i} /(2 \pi)(i=1,2)$ denotes the electrode spatial wavenumber. This has been nondimensionalised as the ratio of the periodicity of the device architecture to the spatial wavelength of the applied voltage. So for example, at fractal generation level $\varrho=3$, each alternate level one ceramic pillar is excited by a voltage that is shifted $180^{\circ}$ out of phase corresponds to $\gamma_{1}=1 / 2$ (see Figure 2 ). 


\subsection{The Fractal Geometry}

The model is configured for periodic 2-2 and 1-3 composites with the main thickness mode of vibration in the $x_{3}$ direction (see Figure 1). By using the periodicity of the structure in the $x_{1}-x_{2}$ plane, the material constants, $M(\underline{x}, \varrho)$, can be expressed as

$$
M(\underline{x}, \varrho)= \begin{cases}\phi, & \text { if } \underline{x} \epsilon S_{\varrho} \\ \theta, & \text { otherwise }\end{cases}
$$

where $\theta$ and $\phi$ are some physical property pertaining to the polymer and the ceramic phase respectively, and $\varrho$ is the fractal generation level. This satisfies the periodicity relationship $M\left(x_{1}+p_{1} a, x_{2}+p_{2} b, \varrho\right)=M\left(x_{1}, x_{2}, \varrho\right), \forall a, b \in \mathbb{Z}$ where $p_{1}$ is the period of the geometry in the $x_{1}$ direction and $p_{2}$ is the period of the geometry in the $x_{2}$ direction. A Fourier series representation for the Sierpinski carpet (1-3 configuration)(see Figure 3(a)) can be written as,

$$
M\left(x_{1}, x_{2}, \varrho\right)=\sum_{m=-\infty}^{\infty} \sum_{n=-\infty}^{\infty} M_{m n}^{\varrho} e^{-\jmath\left(2 \pi m x_{1}+2 \pi n x_{2}\right)}
$$

where the set $S_{\varrho}$ is given by

$$
\begin{gathered}
S_{\varrho}=\bigcup_{q=1}^{\varrho} \bigcup_{i=1}^{8^{q-1}}\left[T^{q}(i, 1), T^{q}(i, 1)+(1 / 3)^{q}\right] \times\left[T^{q}(i, 2), T^{q}(i, 2)+(1 / 3)^{q}\right], \\
T^{1}=\{(-1 / 6,-1 / 6)\}, \\
T^{q}=\bigcup_{i=1}^{8}\left\{\bigcup_{j=1}^{8^{q-2}} \gamma_{i}+\left(T_{j}^{q-1}+\left(\frac{1}{2}, \frac{1}{2}\right)\right) \frac{1}{3}\right\}
\end{gathered}
$$

and $\gamma=\{(1 / 6,1 / 6),(-1 / 6,1 / 6),(-1 / 2,1 / 6),(-1 / 2,-1 / 6),(-1 / 2,-1 / 2),(-1 / 6,-1 / 2),(1 / 6,-1 / 2),(1 / 6,-$ $1 / 6)\} . T^{q}$ corresponds to the co-ordinates of the bottom left hand corner of each ceramic pillar and the translation of $(1 / 2,1 / 2)$ is used to facilitate the contraction of each prefractal in the first quadrant. For the Cantor set geometry (2-2 configuration)(see Figure 3(b)) the Fourier series is expressed as

$$
M(x, \varrho)=\sum_{n=-\infty}^{\infty} M_{n}^{\varrho} e^{-\jmath(2 \pi n x)},
$$

and $S_{\varrho}$ simplifies to

$$
S_{\varrho}=\bigcup_{q=1}^{\varrho} \bigcup_{i=1}^{2^{q-1}}\left[T^{q}(i, 1), T^{q}(i, 1)+(1 / 3)^{q}\right]
$$




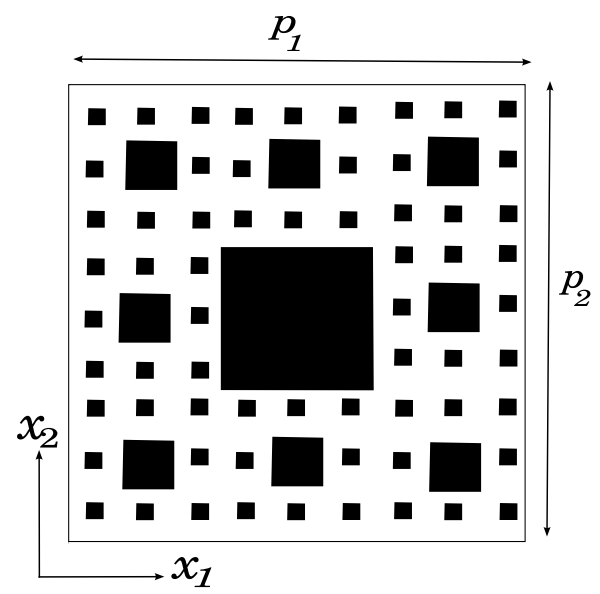

(a)

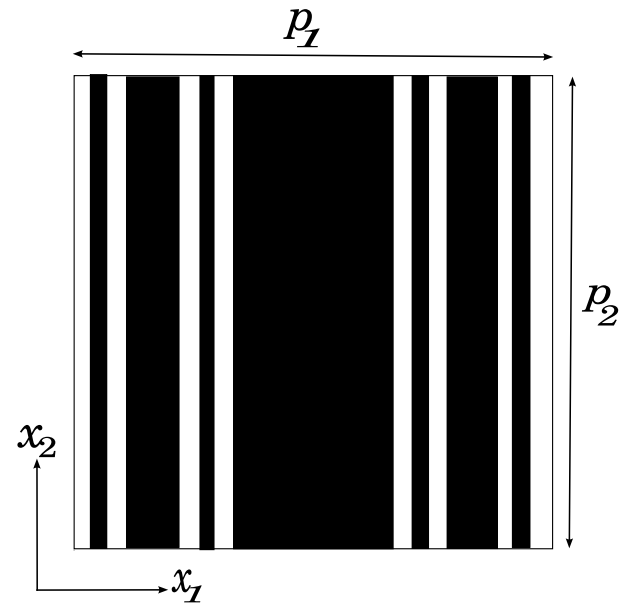

(b)

Figure 3: Plan view of one period of (a) the Sierpinski carpet transducer design, and (b) the Cantor set transducer design. (The black squares represent ceramic pillars and the white material is the polymer. Generation level $\varrho=3$ )

where

$$
T^{q}=\bigcup_{i=1}^{3}\left\{\bigcup_{j=1}^{2^{q-2}} \gamma_{i}+\left(T_{j}^{q-1}+\frac{1}{2}\right) \frac{1}{3}\right\},
$$

$T^{1}=\{-1 / 6\}$ and $\gamma=\{-1 / 2,1 / 6\}$. For the Sierpinski carpet design the Fourier coefficients at fractal generation level $\varrho$ are given by

$$
\begin{aligned}
M_{m n}^{\varrho} & =\frac{(\phi-\theta)}{\pi^{2} m n} \sin \left(\frac{\pi m}{3^{q}}\right) \sin \left(\frac{\pi n}{3^{q}}\right) \\
& \times e^{\left(\jmath \pi m\left(2 T^{q}(j, 1)+(1 / 3)^{q}\right)+\jmath \pi n\left(2 T^{q}(j, 2)+(1 / 3)^{q}\right)\right)} \quad m, n= \pm 1, \pm 2, \ldots \\
M_{0 n}^{\varrho}= & \frac{(\phi-\theta)}{\pi n}\left(\frac{1}{3^{q}}\right) \sin \left(\frac{\pi n}{3^{q}}\right) e^{\left(\jmath \pi n\left(2 T^{q}(j, 2)+(1 / 3)^{q}\right)\right)} \quad n= \pm 1, \pm 2, \ldots, \\
M_{m 0}^{\varrho}= & \frac{(\phi-\theta)}{\pi m}\left(\frac{1}{3^{q}}\right) \sin \left(\frac{\pi m}{3^{q}}\right) e^{\left(\jmath \pi m\left(2 T^{q}(j, 1)+(1 / 3)^{q}\right)\right)} m= \pm 1, \pm 2, \ldots,
\end{aligned}
$$

and

$$
M_{00}^{\varrho}=\frac{\phi-\theta}{3^{2 q}}+\frac{7 \theta}{8 \varrho-1} .
$$

For the Cantor set geometry the Fourier coefficients at fractal generation level $\varrho$ are given by

$$
M_{n}^{\varrho}=\frac{(\phi-\theta)}{\pi n} \sin \left(\frac{\pi n}{3^{q}}\right) e^{\left(\jmath \pi n\left(2 T^{q}(j, 1)+(1 / 3)^{q}\right)\right)} \quad n= \pm 1, \pm 2, \ldots
$$

and

$$
M_{0}^{\varrho}=\frac{\phi-\theta}{3^{q}}+\frac{\theta}{2^{\varrho}-1} .
$$


The double subscript notation can be simplified by ordering the Fourier coefficients. Define the ordered set

$$
H=\{(-N,-M),(-N,-M+1), \ldots,(-N, M),(-N+1,-M), \ldots,(N, M)\}
$$

so that if

$$
G^{s}=\left(\frac{2 \pi}{p_{1}} H^{s, 1}, \frac{2 \pi}{p_{2}} H^{s, 2}, 0\right)
$$

then (7) can be rewritten for a finite number of terms ( $N$ in direction $x_{1}$ and $M$ in direction $x_{2}$ ) as

$$
M\left(x_{1}, x_{2}, \varrho\right)=\sum_{s=1}^{(2 N+1)(2 M+1)} M_{s}^{\varrho} e^{-\jmath G^{s} \cdot r}
$$

where $H^{s, i}$ is the $i^{\text {th }}$ component of element $s$ of $H$. The dependent variables $F(r, t)$ propagating within these periodic structures are then approximated as Floquet series

$$
F(r, t, k, \omega, \varrho)=\sum_{s=1}^{(2 N+1)(2 M+1)} F_{s}^{\varrho}(k, \omega) e^{\jmath\left(\omega t-k \cdot r-G^{s} \cdot r\right)}
$$

where $r=\left(x_{1}, x_{2}, x_{3}\right), t$ is time, $\omega$ is the angular frequency and $k=\left(k_{1}, k_{2}, k_{3}\right)$ is the wave vector. Viscoelastic loss is incorporated into the model by the use of a complex wave vector [12].

\subsection{The Plane Wave Expansion Method}

Denote the generalized displacement field by $u$ where $u=\left(u_{1}, u_{2}, u_{3}, u_{4}=\phi\right)$ and the generalized stress vectors by $t_{i}=\left(T_{i 1}, T_{i 2}, T_{i 3}, D_{i}\right)$. Substituting the expansion (23) into (1) and (2), and equating coefficients, gives

$$
T_{i j}^{p}=\sum_{q=1}^{(2 N+1)(2 M+1)}-\jmath\left(k_{l}+G_{l}^{q}\right)\left(c_{i j k l}^{V p, q} u_{k}^{q}+e_{l i j}^{V^{p, q}} u_{4}^{q}\right)
$$

and

$$
D_{i}^{p}=\sum_{q=1}^{(2 N+1)(2 M+1)}-\jmath\left(k_{l}+G_{l}^{q}\right)\left(e_{i k l}^{V^{p, q}} u_{k}^{q}-\epsilon_{i l}^{V^{p, q}} u_{4}^{q}\right)
$$

where the particular Floquet series component is given by 


$$
V^{p, q}= \begin{cases}p+\frac{(2 N+1)(2 M+1)+1}{2}-q, & \text { if } 1 \leq p+\frac{(2 N+1)(2 M+1)+1}{2}-q \leq(2 N+1)(2 M+1) \\ & \text { and }\left|H^{p, 1}-H^{q, 1}\right| \leq N\left|H^{p, 2}-H^{q, 2}\right| \leq M \\ 0, & \text { otherwise }\end{cases}
$$

and $M^{V^{p, q}}=0$ if $V^{p, q}=0$. In terms of the generalized stress vectors (24) and (25) give

$$
\jmath t_{i}^{p}=\sum_{q=1}^{(2 N+1)(2 M+1)}\left(k_{l}+G_{l}^{q}\right) A_{i l}^{p, q} u^{q}
$$

where

$$
A_{i l}^{p, q}=\left[\begin{array}{cccc}
c_{i 11 l}^{V^{p, q}} & c_{i 12 l}^{V^{p, q}} & c_{i 13 l}^{V^{p, q}} & e_{l i 1}^{V^{p, q}} \\
& & & \\
c_{i 21 l}^{V^{p, q}} & c_{i 22 l}^{V^{p, q}} & c_{i 23 l}^{V^{p, q}} & e_{l i 2}^{V^{p, q}} \\
& & & \\
c_{i 31 l}^{V^{p, q}} & c_{i 32 l}^{V^{p, q}} & c_{i 33 l}^{V^{p, q}} & e_{l i 3}^{V^{p, q}} \\
& & & \\
e_{i 1 l}^{V^{p, q}} & e_{i 2 l}^{V^{p, q}} & e_{i 3 l}^{V^{p, q}} & -\epsilon_{i l}^{V^{p, q}} \\
& & &
\end{array}\right] .
$$

The same analysis can be carried out for equations (3) and (4) to obtain the expression

$$
\left(k_{i}+G_{i}^{p}\right) j t_{i}^{p}=\sum_{q=1}^{(2 N+1)(2 M+1)} \omega^{2} R^{p, q} u^{q}
$$

where

$$
R^{p, q}=\left[\begin{array}{cccc}
\rho^{V^{p, q}} & 0 & 0 & 0 \\
0 & \rho^{V^{p, q}} & 0 & 0 \\
0 & 0 & \rho^{V^{p, q}} & 0 \\
0 & 0 & 0 & 0
\end{array}\right]
$$


Now let $T_{i}=\left[t_{i}^{1}, \ldots, t_{i}^{p}, \ldots, t_{i}^{(2 N+1)(2 M+1)}\right]^{T}, U=\left[u^{1}, \ldots, u^{q}, \ldots, u^{(2 N+1)(2 M+1)}\right]^{T}$,

$$
A_{i j}=\left[\begin{array}{cclc}
A_{i j}^{1,1} & A_{i j}^{1,2} & \ldots & A_{i j}^{1,(2 N+1)(2 M+1)} \\
A_{i j}^{2,1} & A_{i j}^{2,2} & \ldots & A_{i j}^{2,(2 N+1)(2 M+1)} \\
\vdots & \vdots & \ddots & \vdots \\
A_{i j}^{(2 N+1)(2 M+1), 1} & A_{i j}^{(2 N+1)(2 M+1), 2} & \ldots & A_{i j}^{(2 N+1)(2 M+1),(2 N+1)(2 M+1)}
\end{array}\right]
$$

and

$$
R=\left[\begin{array}{cccc}
R^{1,1} & R^{1,2} & \ldots & R^{1,(2 N+1)(2 M+1)} \\
R^{2,1} & R^{2,2} & \ldots & R^{2,(2 N+1)(2 M+1)} \\
\vdots & \vdots & \ddots & \vdots \\
R^{(2 N+1)(2 M+1), 1} & R^{(2 N+1)(2 M+1), 2} & \ldots & R^{(2 N+1)(2 M+1),(2 N+1)(2 M+1)}
\end{array}\right] .
$$

Equations (27) and (29) can then be written compactly as

$$
\jmath T_{i}=A_{i j} \Gamma_{j} U
$$

and

$$
\omega^{2} R U=\Gamma_{i}\left(\jmath T_{i}\right)
$$

where

$$
\Gamma_{i}=\left[\begin{array}{cccc}
\left(k_{i}+G_{i}^{1}\right) I_{4} & 0 & \ldots & 0 \\
0 & \left(k_{i}+G_{i}^{2}\right) I_{4} & \ldots & 0 \\
\vdots & \vdots & \ddots & \vdots \\
0 & 0 & \ldots & \left(k_{i}+G_{i}^{(2 N+1)(2 M+1)}\right) I_{4}
\end{array}\right] .
$$

Equations (33) and (34) can be combined to give the generalised eigenvalue problem

$$
\left[\begin{array}{cc}
\omega^{2} R-B & 0 \\
-C_{2} & I
\end{array}\right]\left(\begin{array}{c}
U \\
J T_{3}
\end{array}\right)=k_{3}\left[\begin{array}{cc}
C_{1} & I \\
D & 0
\end{array}\right]\left(\begin{array}{c}
U \\
\jmath T_{3}
\end{array}\right)
$$

in the $8(2 N+1)(2 M+1)$ eigenvalues $k_{3}^{(r)}$ and corresponding eigenvectors $\left[\begin{array}{c}U \\ j T_{3}\end{array}\right]^{(r)}$ where $B=\sum_{i, j=1,2} \Gamma_{i} A_{i j} \Gamma_{j}, C_{1}=\sum_{i=1,2} \Gamma_{i} A_{i 3}, C_{2}=\sum_{j=1,2} A_{3 j} \Gamma_{j}$ and $D=A_{33}$. Solving equation (35) and introducing the relative amplitudes $A^{(r)}$ gives 


$$
\left(\begin{array}{c}
u(r, t) \\
t_{3}^{q}(r, t)
\end{array}\right)=e^{\jmath\left(\omega t-k_{1} x_{1}-k_{2} x_{2}\right)} \sum_{q=1}^{(2 N+1)(2 M+1)} e^{-\jmath G^{q} \cdot r}\left(\sum_{r=1}^{8(2 N+1)(2 M+1)} A^{(r)} e^{-\jmath k_{3}^{(r)} x_{3}}\left[\begin{array}{l}
u^{q} \\
t_{3}^{q}
\end{array}\right]^{(r)}\right)
$$

Energy distribution within the transducer can be used to clarify particular types of modes in conjunction with examining profiles of the displacements, stresses and electric potential. The energy distribution in the device can be examined using the Poynting vector, defined as

$$
P_{j}=-T_{i j} u_{i, t}+\phi D_{j, t} .
$$

Substituting equations (1) and (2) into equation (37) gives

$$
P_{j}=-\jmath \omega\left(c_{i j k l} u_{k, l}+e_{l i j} \phi_{, l}\right) u_{i}+\jmath \omega \phi\left(e_{j k l} u_{k, l}-\epsilon_{j l} \phi_{, l}\right) .
$$

\subsection{Applying the Boundary Conditions and the Derivation of the Electrical Operating Characteristics}

Applying the mechanical boundary condition of a stress free plate gives, from equation $(36)$

$$
0=\sum_{r=1}^{8(2 N+1)(2 M+1)} A^{(r)} e^{-\jmath k_{3}^{(r)} h}\left(T_{3 i}^{q}\right)^{(r)}, \quad q=1, \ldots,(2 N+1)(2 M+1)
$$

at the top surface $x_{3}=h$ and

$$
0=\sum_{r=1}^{8(2 N+1)(2 M+1)} A^{(r)}\left(T_{3 i}^{q}\right)^{(r)}, \quad q=1, \ldots,(2 N+1)(2 M+1)
$$

at the lower surface $x_{3}=0$. Setting the electrical potential given by equation (5) at $x_{3}=h$ gives, from equation (36)

$$
\begin{aligned}
\sum_{r=1}^{8(2 N+1)(2 M+1)} A^{(r)} \phi^{q,(r)} e^{-\jmath k_{3}^{(r)} h} & =V_{0} \operatorname{sinc}\left(\left(k_{1}+G_{1}^{q}\right) \frac{p_{1}}{2}\right) \\
& \times \operatorname{sinc}\left(\left(k_{2}+G_{2}^{q}\right) \frac{p_{2}}{2}\right), q=1, \ldots(2 N+1)(2 M+1)
\end{aligned}
$$


and at $x_{3}=0$ (with zero electrical potential)

$$
\sum_{r=1}^{8(2 N+1)(2 M+1)} A^{(r)} \phi^{q,(r)}=0 .
$$

Equations (39), (40), (41) and (42) constitute $8(2 N+1)(2 M+1)$ equations in the $8(2 N+$ 1) $(2 M+1)$ unknowns $A^{(r)}$. Hence this system of linear equations can be solved for the coefficients $A^{(r)}$; these are needed below in equation (43) to calculate the electrical admittance and in equation (36) to calculate the displacements, stresses etc. The electrical operating characteristics of the device are then used to examine its resonant behaviour. The admittance $(Y)$ expresses the ease with which an alternating current flows through the transducer and the resonant modes are signified by maxima in the real part of the admittance (the conductance $Y$ ). Using continuity of the electrical potential at the front interface it can be shown that [11]

$$
\begin{gathered}
Y\left(k_{1}, k_{2}, \omega\right)=\jmath \omega \sum_{q=1}^{(2 N+1)(2 M+1)}\left[\sum_{r=1}^{8(2 N+1)(2 M+1)} A^{(r)}\left(D_{3}^{q,(r)}-\epsilon_{0}|\kappa| \phi^{q,(r)}\right) e^{-j k_{3}^{(r)} h}\right] \\
\times p_{1} \operatorname{sinc}\left(\left(k_{1}+G_{1}^{q}\right) \frac{p_{1}}{2}\right) p_{2} \operatorname{sinc}\left(\left(k_{2}+G_{2}^{q}\right) \frac{p_{2}}{2}\right)
\end{gathered}
$$

where $\kappa=\sqrt{\left(k_{1}+G_{1}^{q}\right)^{2}+\left(k_{2}+G_{2}^{q}\right)^{2}}$.

\section{Theoretical Model Results}

In section 3.2 the admittance $(Y)$ and electrical impedance $\left(Z_{T}=1 / Y\right)$ characteristics of a Cantor set composite transducer, with a standard hardset passive phase and PZT5H ceramic (see Tables 1 and 2 for material properties ) are investigated. The effects of the fractal generation level will be discussed and a modal analysis will be performed to categorise any additional modes that arise due to the fractal geometry. In section 3.3 the electrical impedance and admittance characteristics of a Sierpinski carpet composite transducer using these materials is similarly investigated. The lateral spatial periodicity is set as $p_{1}=p_{2}=1 \mathrm{~mm}$ and the thickness of the device is also $h=1 \mathrm{~mm}$. 


\begin{tabular}{|c|c|c|}
\hline Parameter & Symbol/Units & Value \\
\hline Shear modulus (real part) & $G^{\prime}\left(\mathrm{kg} \mathrm{m}^{-1} \mathrm{~s}^{-2}\right)$ & $1.57 \times 10^{9}$ \\
Young's modulus (real part) & $Y^{\prime}\left(\mathrm{kg} \mathrm{m}^{-1} \mathrm{~s}^{-2}\right)$ & $4.28 \times 10^{9}$ \\
Shear Velocity & $c_{G}\left(\mathrm{~m} \mathrm{~s}^{-1}\right)$ & $1.17 \times 10^{3}$ \\
Longitudinal Velocity & $c_{Y}\left(\mathrm{~m} \mathrm{~s}^{-1}\right)$ & $2.51 \times 10^{3}$ \\
Density & $\rho\left(\mathrm{kg} \mathrm{m}^{-3}\right)$ & $1.15 \times 10^{3}$ \\
Dielectric constant & $\epsilon(-)$ & 4 \\
Frequency of measurement & $f_{I}(\mathrm{~Hz})$ & $5.00 \times 10^{5}$ \\
tan $\delta$ frequency maximum & $f_{\max }(\mathrm{Hz})$ & $3.15 \times 10^{5}$ \\
G Attenuation Coefficient & $\alpha_{G}^{0}(N p / m)$ & $41^{b}$ \\
Y Attenuation Coefficient & $\alpha_{Y}^{0}(N p / m)$ & $16^{b}$ \\
\hline
\end{tabular}

Table 1: Physical properties of the polymer phase HY1300/CY1301 Hardset [20].

\begin{tabular}{|c|c|c|c|}
\hline- & Constant & Units & Value \\
\hline elastic constant & $c_{11}$ & $\mathrm{Nm}^{-2}$ & $12.72 \times 10^{10}$ \\
elastic constant & $c_{12}$ & $\mathrm{Nm}^{-2}$ & $8.02 \times 10^{10}$ \\
elastic constant & $c_{13}$ & $\mathrm{Nm}^{-2}$ & $8.47 \times 10^{10}$ \\
elastic constant & $c_{33}$ & $\mathrm{Nm}^{-2}$ & $11.74 \times 10^{10}$ \\
dielectric constant & $\epsilon_{33}$ & - & $1.70 \times 10^{3}$ \\
dielectric constant & $\epsilon_{11}$ & - & $1.47 \times 10^{3}$ \\
Loss Tangent & $\tan \delta$ & - & $1 / 65$ \\
density & $\rho_{b}$ & $\mathrm{~kg} \mathrm{~m}^{-3}$ & $7.50 \times 10^{3}$ \\
Piezoelectric stress coefficient & $e_{33}$ & $\mathrm{C} \mathrm{m}^{-2}$ & 23.30 \\
Piezoelectric stress coefficient & $e_{31}$ & $\mathrm{C} \mathrm{m}$ & -6.50 \\
\hline
\end{tabular}

Table 2: Physical properties of the ceramic phase PZT5H [21].

\subsection{Numerical Implementation}

The model has been implemented in a FORTRAN code that calls NAG [22] subroutines to numerically solve the large matrix equations. In particular they are used to solve 
the system of linear equations (39), (40), (41) and (42) for the mode amplitudes $A^{(r)}$. The matrix of coefficients in these equations ( $X$ say) has dimensions $8(2 N+1)(2 M+$ $1) \times 8(2 N+1)(2 M+1)$ and is fully populated, where $N$ and $M$ are the number of Fourier coefficients used to approximate the device architecture in each lateral direction. Unfortunately the matrix $X$ is ill-conditioned [23]. To help obviate this problem the matrix entries are balanced by scaling the parameters of the model (see Table 3). Each of the parameters is made $O(1)$ by a judicious choice of the scalings $\alpha, \beta, \gamma$ and $\varphi$ so that five equations in four unknowns must be satisfied. This is done by scaling the thickness $h$ by specifying $\beta$, scaling the density $\rho$ by specifying $\alpha$, scaling the piezoelectric stress tensor $e_{i j k}$ by specifying $\varphi$, scaling the elasticity tensor $c_{i j k l}$ by specifying $\gamma$ and this results in an appropriate scaling for the permittivity tensor $\epsilon_{i j}$. The exponential terms

\begin{tabular}{|c|c|c|c|}
\hline Parameter & Units & Dimensions & Scaling \\
\hline$c_{i j k l}$ & $N m^{-2}$ & $M L^{-1} T^{-2}$ & $\alpha \beta^{-1} \gamma^{-2}$ \\
$\epsilon_{i j}$ & $F m^{-1}$ & $C^{2} M^{-1} T^{2} L^{-3}$ & $\phi^{2} \alpha^{-1} \gamma^{2} \beta^{-3}$ \\
$e_{i j k}$ & $C m^{-2}$ & $C L^{-2}$ & $\phi \beta^{-2}$ \\
$\rho$ & $k g m^{-1}$ & $M L^{-1}$ & $\alpha \beta^{-1}$ \\
$h$ & $\mathrm{~m}$ & $\mathrm{~L}$ & $\beta$ \\
\hline
\end{tabular}

Table 3: Dimensions and scaling parameters for the material properties.

that arise when calculating the boundary conditions at $x_{3}=h$ also adversely affect the conditioning of the matrix $X$. To alleviate this problem the rows of $X$ that arise from these boundary conditions are multiplied by a scale factor given by $e^{\max _{r}\left(k^{\prime \prime}{ }_{3}^{(r)}\right) h}$, where $k_{3}^{\prime \prime(r)}$ is the imaginary part of the wavenumber $k_{3}^{(r)}$. The remaining ill-conditioning is then dealt with by the use of Tikhonov regularisation [24]. This involves the introduction of a small parameter $(\mu)$ that shifts the eigenvalues of the matrix $X$ away from zero along the positive real axis. In order to do this the matrix $X$ is multiplied by its complex conjugate $X^{*}$ so that the matrix is real and symmetric and its eigenvalues become real and non-negative. A small real number $\mu$ is then added to the diagonal terms so that the eigenvalues are translated in the positive direction. So the system of equations that 
is ultimately solved is

$$
\left(X^{*} X+\mu I\right) A^{(r)}=X^{*} Q
$$

where the vector $Q$ corresponds to the right hand sides of the system of equations (39), (40), (41) and (42). As the number of Fourier coefficients increases the implementation time of the model also increases. In the following results, fifteen Fourier coefficients are used to approximate the geometry of the transducer. For the Cantor Set design the matrix dimensions are then roughly $400 \times 400$ and for the Sierpinski Carpet the dimensions are approximately $20000 \times 20000$. The results shown below were produced on a standard desktop computer; the longest computation was for Figure 19 and this took approximately three hours of CPU time. As the fractal generation level increases the number of Fourier coefficients required to represent the fine structure of such a device grows exponentially and therefore so does the computation time. However, from practical manufacturing considerations, the number of generation levels will be limited to three or four, and so the methodology presented here is appropriate.

\subsection{The Cantor Set Transducer}

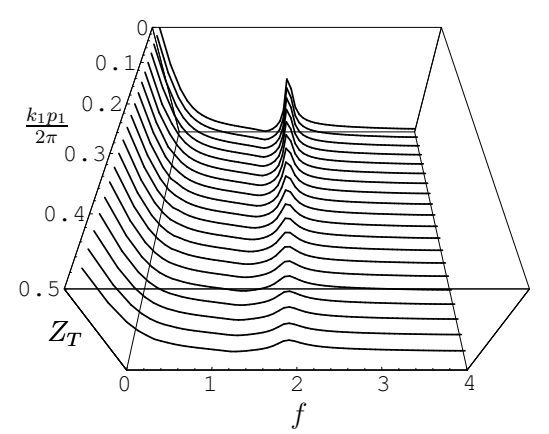

(a)

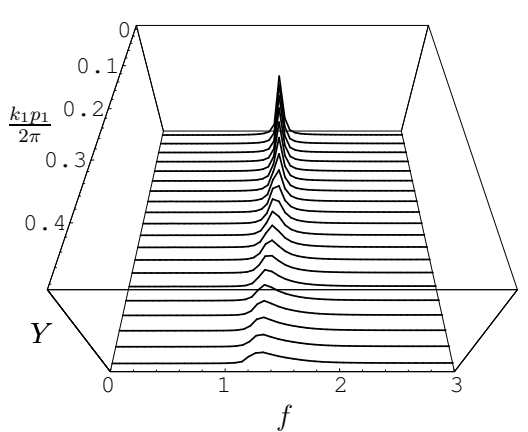

(b)

Figure 4: The absolute value of (a) the electrical impedance $Z_{T}$ (normalised) and (b) the conductance $Y$ (normalised) plotted against the nondimensionalised electrode wavenumber $k_{1} p_{1} / 2 \pi$ and the driving frequency $f(\mathrm{MHz})$ with fractal generation level $\varrho=1$ for the Cantor set transducer.

By examining the impedance profile in Figure 4 (a), the mechanical resonant fre- 
quency $\left(f_{m}\right)$ is identified as the central ridge at around $2 \mathrm{MHz}$ and the electrical resonant frequency $\left(f_{e}\right)$ as the minima at around 1.5 MHz. The electrical resonant frequency corresponds to the thickness mode; a piston-like motion of the device in the $x_{3}$ direction. To gauge the effect of the fractal geometry on the behaviour of the device a comparison with a one dimensional effective medium model (Linear Systems Model (LSM) [25])is conducted. Fractal generation level $\varrho=1$ corresponds to the regular design shown in Figure 1(b). Comparison to the LSM model in Figure 5 shows that there is good agreement between both methods in the location of the modes $\left(f_{m}=1.9 \mathrm{MHz}\right)$ however the PWE model predicts that the magnitude of the thickness mode response will be slightly smaller. As the top electrode spacing varies from $\gamma_{1}=0$ (that is a single, infinitely long top electrode) to $\gamma_{1}=1 / 2$ (see Figure 2 ), there is very little change in the profile (see Figure 4 ).

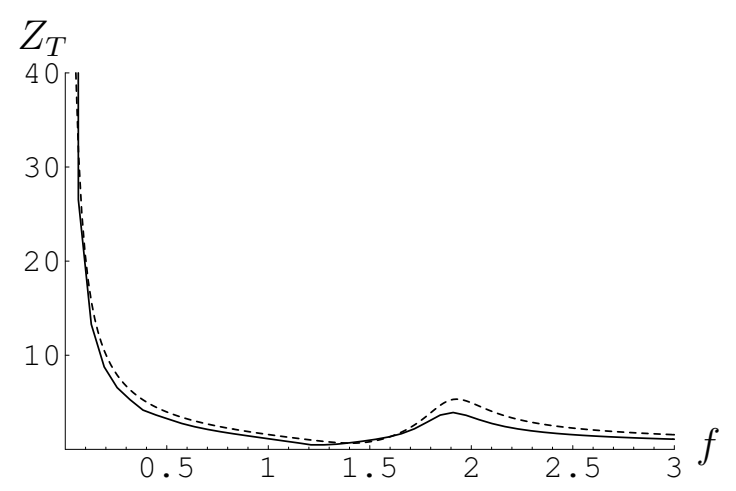

Figure 5: Absolute value of the electrical impedance $Z_{T}(k \Omega)$ against frequency $f$ (Hertz) $\times 10^{6}$ using the LSM method (dashed line) and the fractal PWE method (solid line) with fractal generation level $\varrho=1$ for the Cantor set transducer $\left(\gamma_{1} \approx 0\right)$. 


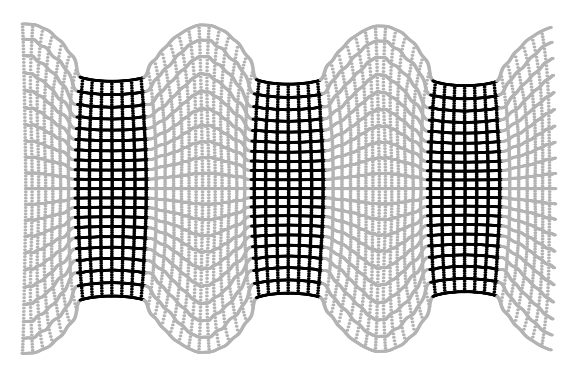

(a)

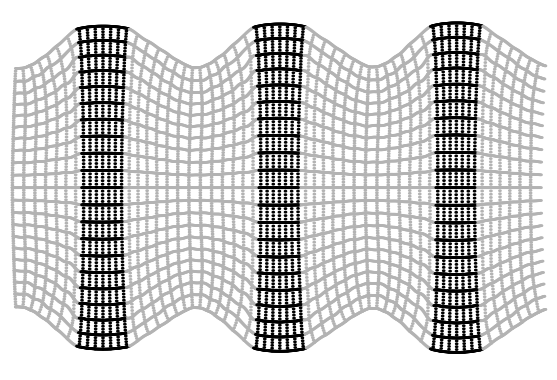

(b)

Figure 6: The real part of the in-plane displacement in the $x_{3}-x_{1}$ plane $x_{2}=0\left(f_{e}=1.46\right.$ $\mathrm{MHz}$ and $\varrho=1)$. Plot (a) is at time $t_{0}$ and $(\mathrm{b})$ is at time $t_{0}$ plus half the period (the displacements have been scaled to accentuate the motion, the dark area is the ceramic and the lighter area is the polymer). The electrical stimulus from the top electrode has a wavenumber of $k_{1}=157 \mathrm{~m}^{-1}$ which corresponds to essentially a single electrode covering all the ceramic pillars.

By investigating the displacement in the $x_{3}-x_{1}$ plane $x_{2}=0$ in Figure 6 it can be seen that at this frequency the transducer is moving in a piston-like fashion with very little motion in the $x_{1}$ direction, and the ceramic pillars move out of phase with the polymer. This is the thickness mode since the mode is symmetric, $u_{1}$ is negligible compared to $u_{3}$ and the amplitude of the displacement is large. By examining the Poynting vector in the same plane in Figure 7 it can be seen that the energy is distributed across the transducer in the $x_{3}$ direction but mainly in the ceramic phase. 


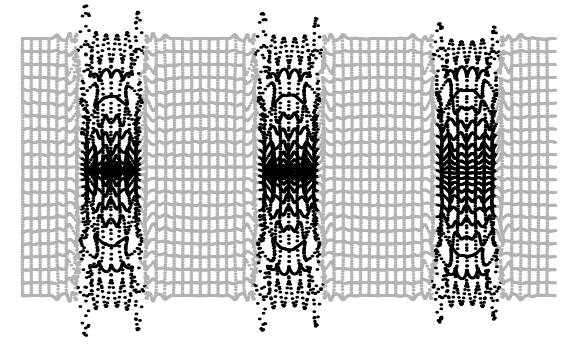

(a)

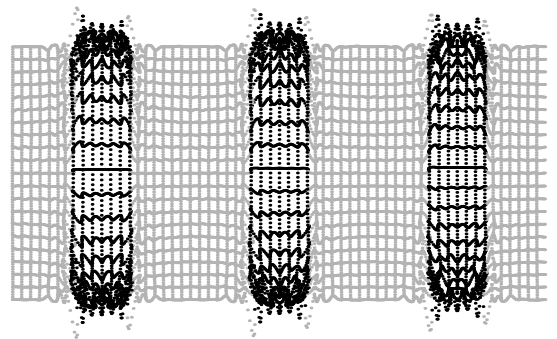

(b)

Figure 7: The real part of the Poynting in the $x_{3}-x_{1}$ plane $x_{2}=0\left(k_{1}=157 \mathrm{~m}^{-1}, f_{e}=1.46\right.$ $\mathrm{MHz}$ and $\varrho=1$ ). Plot (a) is at time $t_{0}$ and (b) is at time $t_{0}$ plus half the period (the Poynting vector has been scaled to accentuate the motion, the dark area is the ceramic and the lighter area is the polymer).

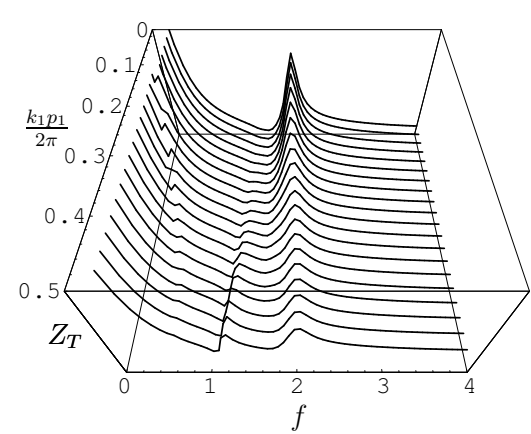

(a)

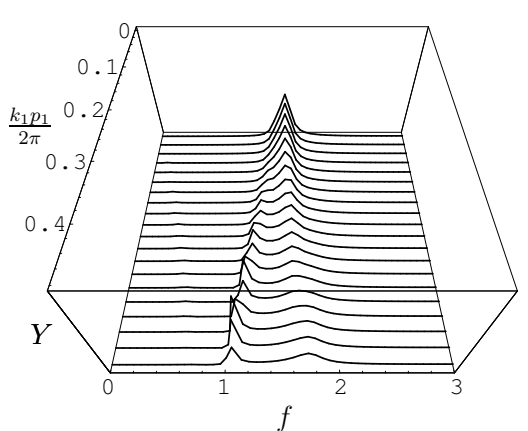

(b)

Figure 8: The absolute value of (a) the electrical impedance $Z_{T}$ and (b) the conductance $Y$ plotted against the nondimensionalised electrode wavenumber $k_{1} p_{1} / 2 \pi$ and the driving frequency $f(\mathrm{MHz})$ for fractal generation level $\varrho=2$ for the Cantor set transducer.

Figure 8 shows the absolute value of the electrical impedance and conductance of the transducer as a function of the driving frequency and the nondimensionalised wavenumber $k_{1} p_{1} / 2 \pi$ for fractal generation level two. It is found that as the spatial wavelength of the electrical excitation decreases (as $k_{1} p_{1} / 2 \pi$ increases), an additional mode is introduced. When $k_{1} p_{1} / 2 \pi$ is small the top electrode acts as a single electrode, essentially treating the device as a homogeneous medium. As $k_{1} p_{1} / 2 \pi$ increases, the heterogeneities in the 
medium start to affect its behaviour.

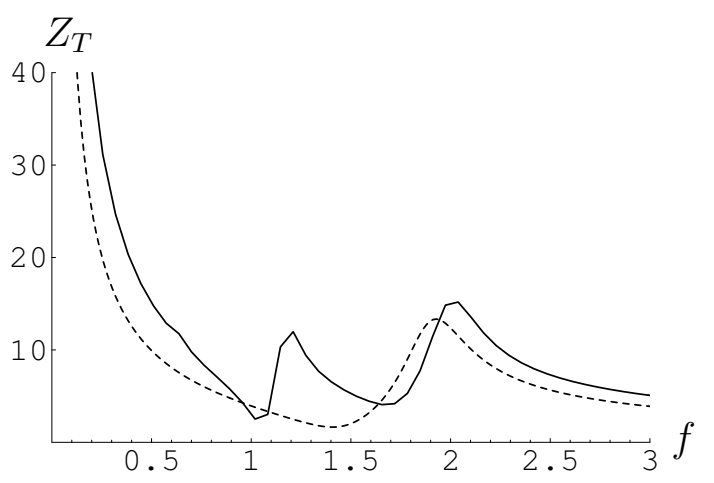

Figure 9: Absolute value of the electrical impedance $Z_{T}(k \Omega)$ against frequency $f$ (Hertz) $\times 10^{6}$ using the LSM method (dashed line) and the fractal PWE method (solid line) for fractal generation level $\varrho=2\left(\gamma_{1}=1 / 2\right)$.

Comparison with the LSM model in Figure 9 suggests that the mechanical resonant frequency (of the homogeneous device) is the second peak at $2 \mathrm{MHz}$.

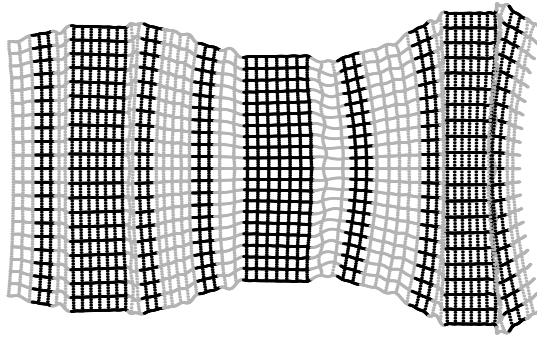

(a)

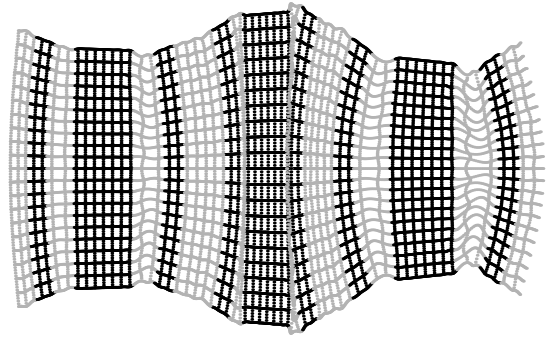

(b)

Figure 10: The real part of the in-plane displacement in the $x_{3}-x_{1}$ plane $x_{2}=0\left(k_{1}=2983\right.$ $\mathrm{m}^{-1}, f_{e}=1.08 \mathrm{MHz}$ and $\varrho=2$ ). Plot (a) is at time $t_{0}$ and (b) is at time $t_{0}$ plus half the period (the displacements have been scaled to accentuate the motion). Here the wavenumber for the electrical excitation corresponds to each alternate generation level 1 ceramic pillar being phase opposed $\left(\gamma_{1}=1 / 2\right.$ see Figure 2$)$.

By investigating the real part of the in-plane displacement in the $x_{3}-x_{1}$ plane $x_{2}=0$ in Figure 10 for the lower frequency mode $\left(f_{e}=1.08 \mathrm{MHz}\right)$ it is found that the alternate level $\varrho=1$ pillars are out of phase and the level $\varrho=2$ pillars, which arise from the new 
fractal generation level, are in phase with their neighbouring level $\varrho=1$ pillar. The spatial periodicity in the electrical excitation $\left(k_{1}=2983 \mathrm{~m}^{-1}\right)$ corresponds to the generation level $\varrho=1$ pillars being spaced at half the wavelength of this excitation. This accounts for the adjacent level $\varrho=1$ pillars being phase opposed. The motion at the top and bottom of the transducer is predominantly in the $x_{3}$ direction, with only lateral motion in the middle of the transducer. As the large pillars become tall and thin, the smaller pillars are squashed inwards and as they become short, the smaller pillars are pushed outwards.

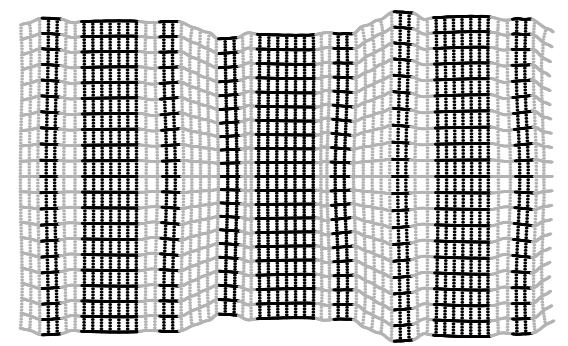

(a)

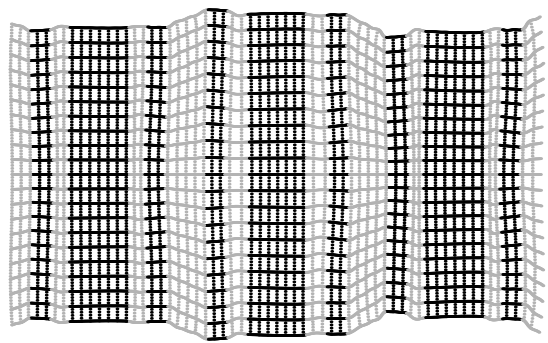

(b)

Figure 11: The real part of the in-plane displacement in the $x_{3}-x_{1}$ plane $x_{2}=0\left(k_{1}=2983\right.$ $\mathrm{m}^{-1}, \gamma_{1}=1 / 2, f_{e}=1.72 \mathrm{MHz}$ and $\varrho=2$ ). Plot (a) is at time $t_{0}$ and (b) is at time $t_{0}$ plus half the period (the displacements have been scaled to accentuate the motion).

Modal analysis of the higher frequency mode (the second minimum in Figure 9 at $f_{e}=1.72 \mathrm{MHz}$ ) also shows a motion that is mainly in the vertical direction. In contrast to the previous case, as the large pillars become tall, the smaller pillars are now pushed outwards. Examination of the Poynting vector indicates that the energy is mainly at the faces of the transducer, particularly at the top face. As $x_{1}$ increases, the damping will increase since the imaginary part of the wavenumber $k_{1}$ is positive. Both of these modes show characteristics of a thickness mode and the presence of two thickness modes has arisen due to the inclusion of the second generation level of ceramic pillars.

This device can therefore transmit over a wider range of frequencies than the regular design. This has practical implications as this will allow electrical excitations composed of a range of frequencies to be used. These broadband coded excitations (such as chirps) lead to improved image resolution in medical applications. The disadvantage is that the peak 
conductance has been reduced and so the efficiency of the device (its ability to convert the electrical energy into mechanical energy in the thickness direction) has been reduced. This is due to the unwanted lateral motion of the pillars that has been introduced, which transmits mechanical energy in the lateral directions. Unlike the regular design, the main peak in the conductance has been shifted to a lower frequency (from around $1.5 \mathrm{MHz}$ to $1 \mathrm{MHz}$ ). So this device also has the ability to switch its main frequency of operation by simply adjusting the electrical input (that is the spatial periodicity of the top electrode excitation). Thus a single device of this type could replace two single frequency devices of standard design. In practical applications a particular transmission frequency is often required because of the resonant behaviour of the system being interrogated.

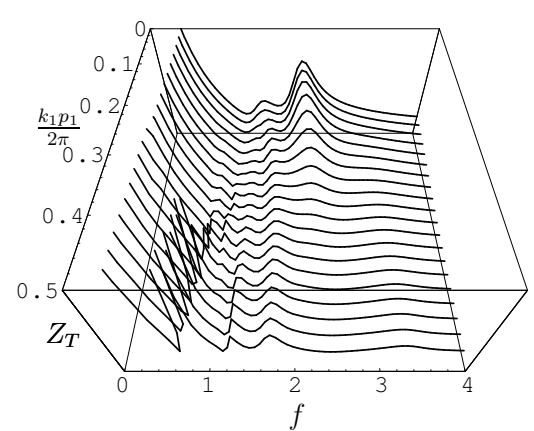

(a)

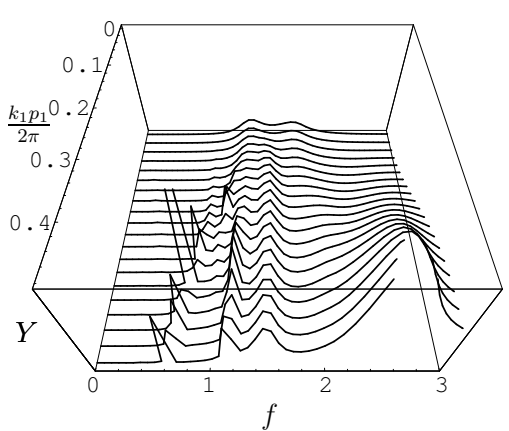

(b)

Figure 12: The absolute value of (a) the electrical impedance $Z_{T}$ and (b) the conductance $Y$ plotted against the nondimensionalised wavenumber $k_{1} p_{1} / 2 \pi$ and the driving frequency $f(\mathrm{MHz})$ for $\varrho=3$ for the Cantor set transducer.

The electrical impedance and conductance of a transducer with fractal generation level $\varrho=3$ is plotted as a function of frequency and the nondimensionalised wavenumber $k_{1} p_{1} / 2 \pi$ in Figure 12. By introducing another fractal generation level, there are now 4 modes present in each plot. There are two additional lower frequency modes within the impedance plot and as the wavelength of the electrical excitation decreases $\left(k_{1} p_{1} / 2 \pi\right.$ increases) the main lobe around $2 \mathrm{MHz}$ is damped out. 


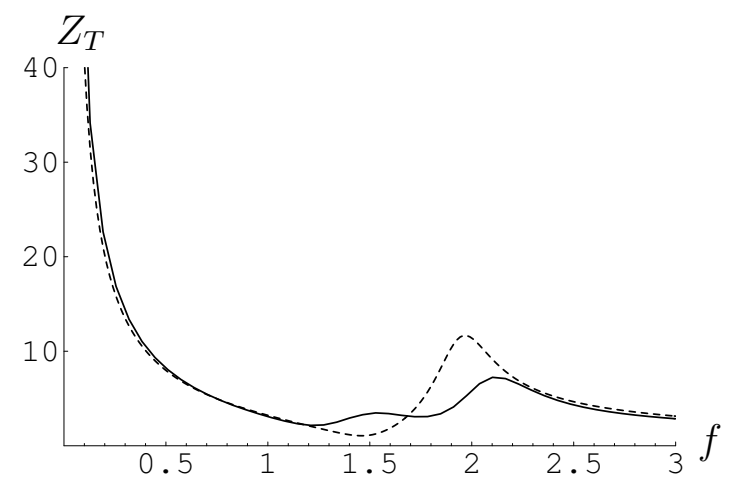

Figure 13: Absolute value of the electrical impedance $Z_{T}(k \Omega)$ against frequency $f$ (Hertz) $\times 10^{6}$ using the LSM method (dashed line) and the fractal PWE method (solid line) with $\varrho=3\left(\gamma_{1} \approx 0\right)$.

Comparison with the LSM method when $\gamma_{1}=k_{1} p_{1} / 2 \pi$ is small shows that there is good agreement away from the resonant regions where there are now two modes present in the PWE method. By analysing the displacements of the first mode in Figure 12 (b) $\left(\gamma_{1}=k_{1} p_{1} / 2 \pi=0.5\right.$ and $\left.f=0.63 \mathrm{MHz}\right)$, it is found that the mode is antisymmetric. The pillars are stretched and squashed at opposite faces of the transducer and the energy is predominantly at the faces of the transducer. This behaviour is symptomatic of an anti-symmetrical Lamb mode, although it has to be borne in mind that this is a pseudodescription, given that the medium is heterogeneous, piezoelectric, anisotropic and lossy. Displacement plots at the second minima in Figure 12(b) show that the large pillars are out of phase from the small pillars and the mode is symmetric. As the large pillars become small the additional pillars are shifted apart in the middle and squashed inwards at the faces of the transducer. The Poynting vector shows that the energy is distributed evenly in the thickness direction of the transducer. Since the displacement is mainly in the $x_{3}$ direction, the mode is classified as a thickness mode. The displacement of the mode occurring around $1.5 \mathrm{MHz}$ in Figure $12(\mathrm{~b})$ is mainly vertical, although the pillars are slightly stretched and squashed at opposite faces. This mode is anti-symmetric, the level $\varrho=1,2$ and 3 neighbouring pillars move as one, and each alternating set of these is $180^{\circ}$ out of phase. Once again, the energy is mainly at the faces, with a large part of the energy distributed within the higher generation level pillars. By investigating the displacements 
of the mode occurring around $2.43 \mathrm{MHz}$ it is found that the overall motion shows the ceramic phase being stretched and squashed, at alternating faces of the transducer (i.e. in a flexural motion). Here the largest pillars are out of phase from the two higher fractal generation level pillars. The energy is distributed along the top face of the transducer and the motion is being driven by the flexural response of the large ceramic pillar and so this mode can be categorised as an intra-pillar mode.

So for larger values of the electrode spacing wavenumber $\left(\gamma_{1}\right)$ the lateral modes interfere with the thickness mode. This mix of flexural modes, guided waves and piston like behaviour will reduce the efficiency of the device. For these electrode spacings this is therefore not a good design. The use of high aspect ratio ceramic pillars (that is long and thin) would help to alleviate this problem. For small values of $\gamma_{1}$ however the device does give rise to a broad conductance distribution, although the amplitude is low. As the fractal generation level increases, the volume fraction of piezoelectric material increases, and this leads to a larger amplitude in the conductance plots.

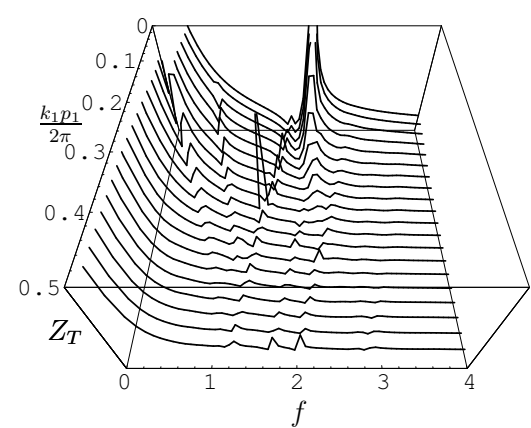

(a)

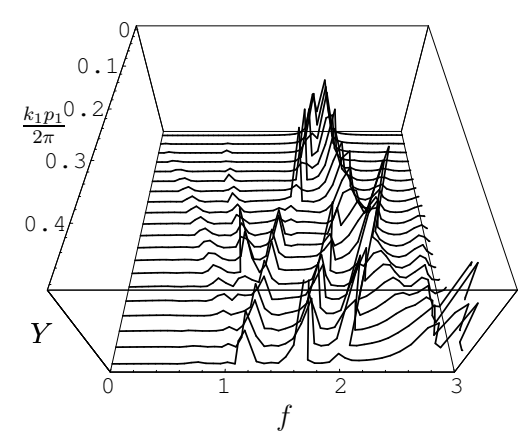

(b)

Figure 14: The absolute value of (a) the electrical impedance $Z_{T}$ and (b) the conductance $Y$ plotted against the nondimensionalised wavenumber $k_{1} p_{1} / 2 \pi$ and the driving frequency $f(\mathrm{MHz})$ for $\varrho=4$ for the Cantor set transducer.

The electrical impedance and conductance of a transducer with four fractal generation levels is plotted as a function of frequency and the nondimensionalised wavenumber $k_{1} p_{1} / 2 \pi$ in Figure 14. By introducing another fractal generation level, there are now 5 modes present in each diagram and as $k_{1} p_{1} / 2 \pi$ increases the main lobe around $2 \mathrm{MHz}$ 
is damped. The results were produced using fifteen Fourier coefficients, which does not fully resolve the generation level four pillars, and is essentially a generation level three simulation with a stiffer polymer phase. These five modes are a complicated mix of flexural, symmetric and guided waves and so the efficiency of this device is far from optimal. The thickness of the device is identical to its lateral periodicity and so the resonant frequency of the (desired) thickness mode harmonic is of a similar magnitude to some of the (unwanted) lateral modes. A thicker design,with high aspect ratio pillars, would help to separate these frequencies and may lead to an improved bandwidth for the thickness mode.

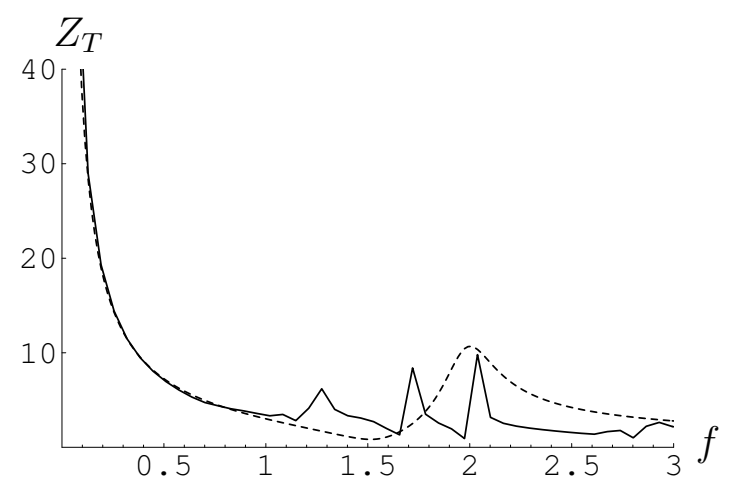

Figure 15: Absolute value of the Electrical impedance $Z_{T}(k \Omega)$ against frequency $f(\mathrm{MHz})$ using the LSM method (dashed line) and the fractal PWE method (solid line) with $\varrho=4$ $\left(\gamma_{1}=1 / 2\right)$.

Comparison to the LSM method shows that both methods predict a mode around 2 MHz. The PWE method predicts up to 5 modes for $\varrho=4$ and the profiles of the modes are less smooth than before. 


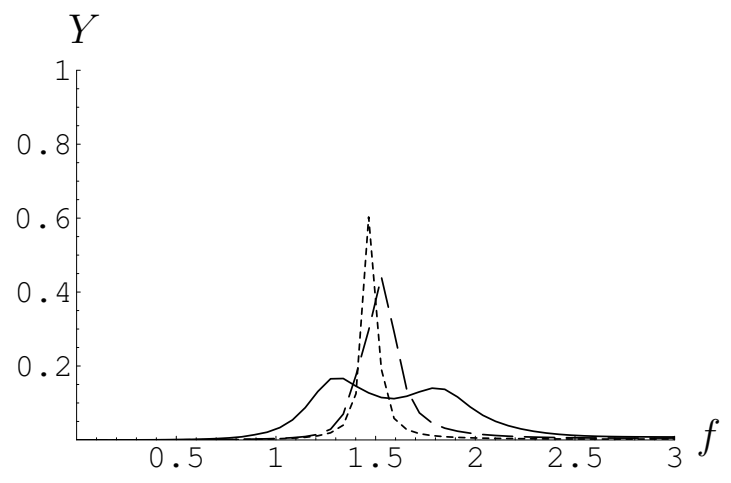

Figure 16: Absolute value of the Electrical conductance $Y(k \Omega)$ against frequency $f$ (Hertz) $\times 10^{6}$ using the fractal PWE method. The small dashed, large dashed and full lines represent fractal generation $\varrho=1, \varrho=2$, and $\varrho=3$ respectively of the Cantor set transducer.

The electrical conductance of the transducer at various fractal generation levels is plotted as a function of frequency in Figure 16. When using only one fractal generation level, the amplitude of the conductance is found to be 0.6 and the $6 \mathrm{~dB}$ percentage bandwidth is $6 \%$. By introducing another fractal generation level $(\varrho=2)$, the amplitude decreases to 0.44 but the bandwidth has increased to $7 \%$. Increasing the fractal generation level to 3 gives rise to a double lobed thickness mode with amplitude 0.17 and bandwidth $65 \%$. The inclusion of extra pillars has increased the bandwidth but as a result the amplitude of the thickness mode has been compromised. These fractal designs have the potential to improve the bandwidth of the transducer as they can introduce additional thickness mode resonances. However, this will require a design with high aspect ratio ceramic pillars, that separates the frequencies of the (desired) thickness modes from the (unwanted) lateral modes.

\subsection{The Sierpinski Carpet Transducer}

In this section the Sierpinksi carpet (1-3 configuration) device shown in Figure 3(a) is investigated. 


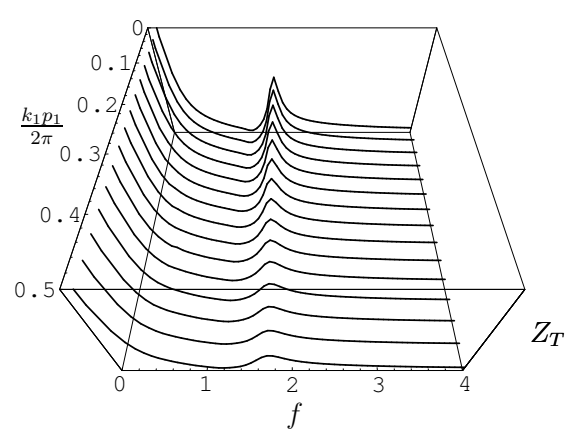

(a)

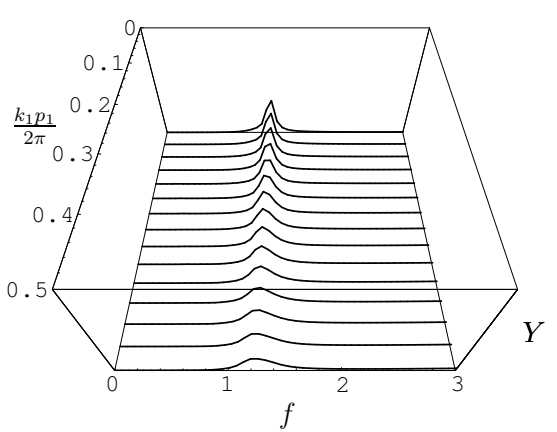

(b)

Figure 17: The absolute value of (a) the electrical impedance $Z_{T}$ and (b) the conductance $Y$ plotted against the nondimensionalised electrode spacing $k_{1} p_{1} / 2 \pi$ and the driving frequency $f(\mathrm{MHz})$ with fractal generation level $\varrho=1$ for the Sierpinski Carpet transducer.

As above, the generation level one device is examined first as this is representative of the designs currently in use. By examining the displacements of the modes shown in the impedance profile in Figure 17 (a), the mechanical resonant frequency $\left(f_{m}\right)$ can be identified as the central ridge at around $1.8 \mathrm{MHz}$ and the electrical resonant frequency $\left(f_{e}\right)$ as the minima at around 1.4 MHz. As the electrode spacing decreases, that is, as $k_{1} p_{1} / 2 \pi$ increases, the main lobes in each plot are attenuated but remain at a fixed frequency.

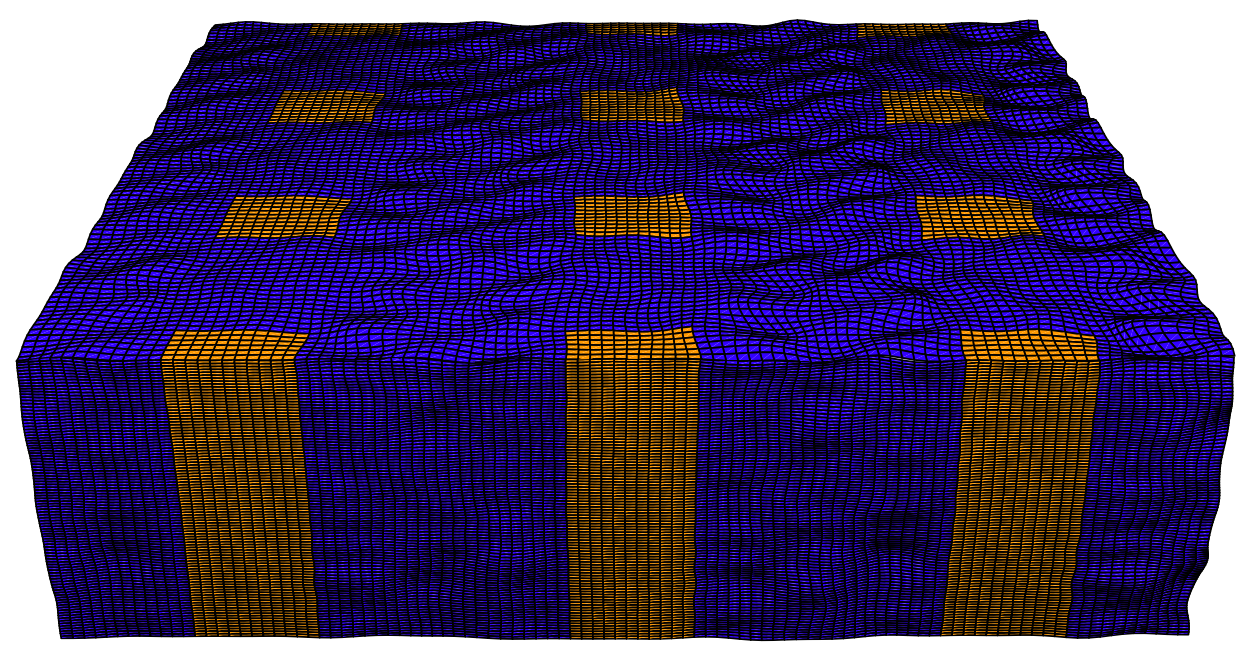

Figure 18: Surface displacement for fractal generation level $\varrho=1$ for the Sierpinski Carpet transducer. 


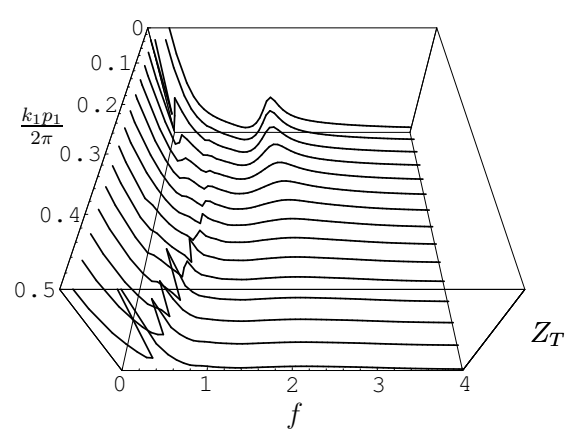

(a)

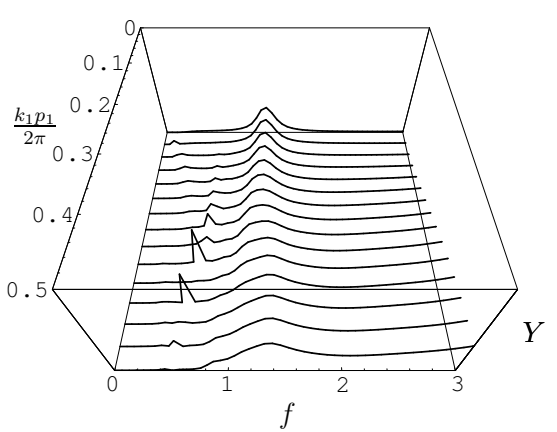

(b)

Figure 19: The absolute value of (a) the electrical impedance $Z_{T}$ and (b) the conductance $Y$ plotted against the nondimensionalised wavenumber $k_{1} p_{1} / 2 \pi$ and the driving frequency $f(\mathrm{MHz})$ for fractal generation level $\varrho=2$ for the Sierpinski Carpet transducer.

Figure 19 shows the absolute value of the electrical impedance and conductance of the transducer as a function of the driving frequency and the nondimensionalised wavenumber $\gamma_{1}=k_{1} p_{1} / 2 \pi$ for fractal generation level two. Once again it is found that by introducing an extra fractal generation level an additional mode is introduced. Examination of the displacement profiles for this mode show it to be an unwanted lateral mode. However, as can be seen in Figure 19(b), as the electrode wavenumber increases the bandwidth of the thickness mode also increases. Comparison with the regular design in Figure 17(b) shows that an improved bandwidth has been achieved with just two generation levels. 


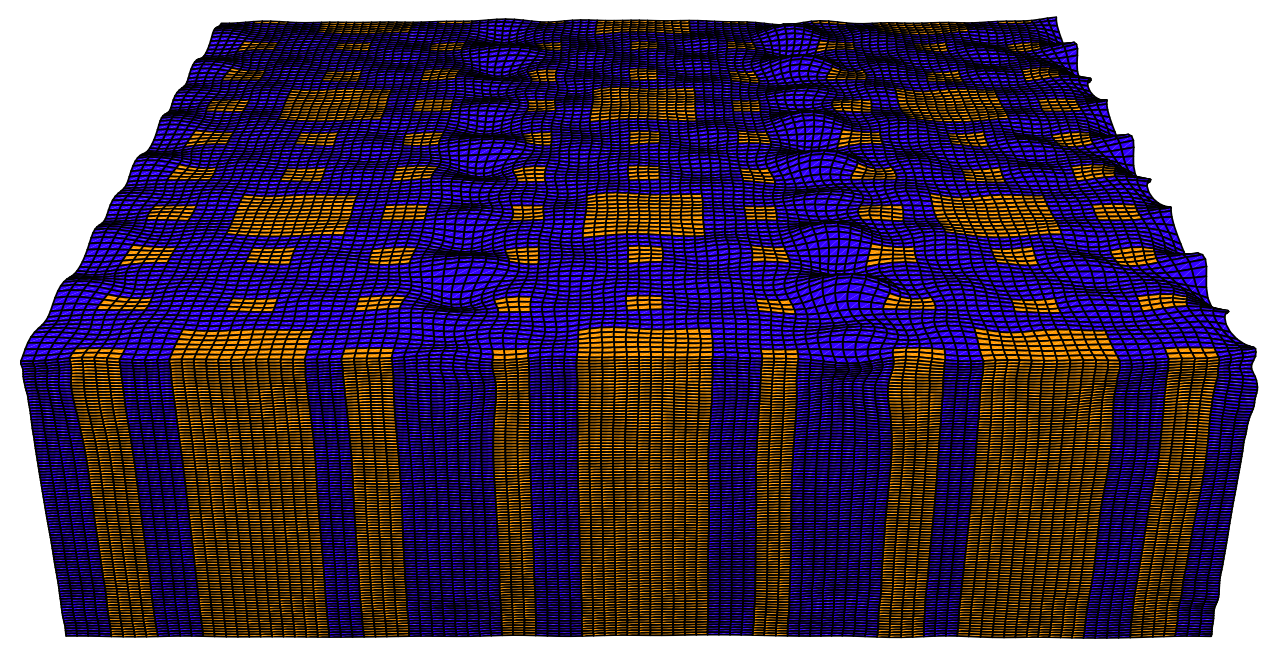

Figure 20: Surface displacement for fractal generation level $\varrho=1$ for the Sierpinski Carpet transducer.

\section{Conclusions}

In general, ultrasonic transducers composed of a periodic piezoelectric composite realise better operational characteristics than single phase designs. The two phase material has reduced mechanical impedance, that better matches the impedance of the mechanical load, and this aids the transfer of mechanical energy into the load material. The most frequently used designs are manufactured by dicing the ceramic into a series of pillars and then filling the void with a passive polymer phase. The architecture of these devices is very regular and has arisen due to manufacturing constraints rather than performance optimisation. However, many of these restrictions no longer hold due to new manufacturing processes such as computer controlled, laser cutting machines, and so there is now freedom to investigate new types of geometry. Hence, in this paper, devices with selfsimilar constructions over a small number of generation levels have been investigated. It is shown that the plane wave expansion model (PWE) can be utilised to investigate the behaviour of these new composite piezoelectric transducers. Of course, from a manufacturing perspective, it will only be possible to build fractal devices over a limited number of generation levels. The effects of introducing up to four fractal generation levels have been 
investigated for a Cantor set geometry transducer and a modal analysis was performed to help explain its characteristics. The results highlight the potential of these designs to broaden the bandwidth of these transducers. Recently, it has been shown that the use of broadband coded excitations such as chirps, has tremendous potential in improving medical image resolution using ultrasound transducers. However this technology requires new transducer designs capable of producing these broadband signals. Such broadband devices would also be of use in second harmonic imaging techniques whereby the received signal is analysed at twice the frequency of the transmitted wave. It was shown in this paper that by increasing the fractal generation level, the bandwidth surrounding the main thickness mode will increase, albeit with a corresponding reduction in the displacement amplitude. The PWE method was also used to investigate the effects of using a transducer with a Sierpinski Carpet geometry. It was found in both fractal architectures (1-3 and 2-2 configurations) that by introducing more fractal generation levels, additional modes will occur which may be able to broaden the operational bandwidth. In addition it was shown that by varying the spatial periodicity of the electrical excitation a shift in the main operating frequency of these devices could be achieved. These preliminary results provide an indication of the potential of these devices. The implementation of the model is sufficiently fast that it could be used to drive a design optimisation routine. The results presented here would no doubt improve if high aspect ratio (long and thin) ceramic pillars were employed to enhance the efficiency of the thickness mode. One disadvantage of the suboptimal designs presented here is the presence of unwanted lateral modes at certain electrode spacings that reduce the amplitude of the thickness mode and ultimately the efficiency of the device in transmitting energy in the thickness direction.

\section{References}

[1] C.J. Harvey, J.M. Pilcher, R.J. Eckersley, M.J.K. Blomley and D.O. Cosgrove, "Advances in Ultrasound", Clin. Rad., vol. 57, no. 3, pp 157-177, Mar. 2002.

[2] K.C. Benjamin, "Recent Advances in 1-3 Piezoelectric Polymer Composite Transducer 
Technology for AUV/UUV Acoustic Imaging Applications", J. Electroceramics, vol. 8, no. 2, pp. 145-154, Aug. 2002.

[3] M. Moles, N. Dube, S. Labbe and E. Ginzel, "Review of Ultrasonic Phased Arrays for Pressure Vessel and Pipeline Weld Inspections", J. Pressure Vessel Technology-Trans. ASME, vol. 127, no. 3, pp. 351-356, Aug. 2005.

[4] G. Hayward and J. Hyslop, "Determination of Lamb Wave Dispersion in Data in Lossy Anisotropic Plates Using Time Domain Finite Element Analysis. Part 1: Theory and Experimental Verification", IEEE Trans. UFFC., vol. 53, no. 2, pp. 443-448, Feb. 2006.

[5] G. Hayward and J. Hyslop, "Determination of Lamb Wave Dispersion in Data in Lossy Anisotropic Plates Using Time Domain Finite Element Analysis. Part 2: Application to 2-2 and 1-3 Piezoelectric Composite Transducer Arrays", IEEE Trans. UFFC., vol. 53, no. 2, pp. 449-455, Feb. 2006.

[6] H.P. Savakus, K.A. Klicker and R.E. Newnham, "PZT-Epoxy Piezoelectric Transducer: A Simplified Fabrication Procedure", Mat. Res. Bull., vol. 16, pp. 677-680, 1981.

[7] C-H. Hu, R. Liu, Q. Zhou, J. Yena and K.K. Shung, "Coded Excitation using Biphasecoded Pulse with Mismatched Filters for High-frequency Ultrasound Imaging.", Ultrasonics, vol. 44, pp. 330-336, 2006.

[8] M.J. Bennett, S. McLaughlin, T. Anderson and N. McDicken, "The use of the Fractional Fourier Transform with Coded Excitation in Ultrasound Imaging.", IEEE Trans. BioMed. Eng., Vol. 53, No. 4, pp. 754-756, 2006.

[9] A. Nowicki, Z. Klimonda, M. Lewandowski, J. Litniewski, P.A. Lewin and I. Trots, "Comparison of Sound Fields Generated by Different Coded Excitations - Experimental Results.", Ultrasonics, vol. 44, pp. 121-129, 2006. 
[10] J. Borsboom, C.T. Chin, A. Bouakaz, M. Versluis and N. de Jong, "Harmonic Chirp Imaging Method for Ultrasound Contrast Agent.", IEEE Trans. UFFC., vol. 52, pp. 241-249, 2005.

[11] M. Wilm, S. Ballandras, V. Laude, and T. Pastureaud, "A Full 3D Plane-WaveExpansion Model for 1-3 Piezoelectric Composite Structures", JASA, vol. 112, no. 3, pp. 943-952, Sept. 2002.

[12] L-A. Orr, A.J. Mulholland, R.L. O'Leary and G. Hayward "Incorporation of Viscoelastic Loss into the Plane Wave Expansion Approach to Modelling Composite Transducers." Proceedings of the 2006 IEEE International Ultrasonics Symposium, pp472-475, 2006.

[13] J. Kigami, "Harmonic Calculus on P.C.F. Self-similar Sets", Trans. AMS, Vol. 335, No. 2, pp. 721-755, 1993.

[14] M.L. Lapidus, "The Vibrations of Fractal Drums and Waves in Fractal Media", Fractals in the Natural and Applied Sciences, Vol. 41, pp. 255-260, 1994.

[15] M. Yamaguti, M. Hata and J. Kigami, J., Mathematics of Fractals, American Mathematical Society, Vol. 167, Rhode Island, USA, 1997.

[16] K. Falconer and J. Hu, "Nonlinear Diffusion Equations on Unbounded Fractal Domains", J Math Anal App, Vol. 256, pp. 606-624, 2001.

[17] J. Abdulbake, A.J. Mulholland and J. Gomatam, "A Renormalisation Approach to Reaction-Diffusion Processes on Fractals.", Fractals, 11(4), pp315-330, 2003.

[18] J. Abdulbake, A.J. Mulholland and J. Gomatam, "Existence and Stability of Reaction-Diffusion Waves on a Fractal Lattice.", Chaos, Solitons and Fractals, 20(4), pp. $799-814,2003$.

[19] "ANSI/IEEE Standard on Piezoelectricity", IEEE Trans. UFFC., vol. 43, no. 5, pp. 717-771, Sept. 1996. 
[20] R.L. O'Leary, G. Smillie, G. Hayward and A.C.S. Parr, CUE Materials Database, Centre for Ultrasonic Engineering, University of Strathclyde, Glasgow, Scotland, June 2002, www.cue.ac.uk.

[21] Ferroperm UK Ltd, Vauxhall Industrial Estate, Ruabon, Wrexham, United Kingdom, LL14 6HA, www.ferroperm.co.uk.

[22] The Numerical Algorithms Group Ltd, Wilkinson House, Jordan Hill Road, Oxford OX2 8DR, www.nag.co.uk.

[23] M.J.S. Lowe, "Matrix Techniques for Modeling Ultrasonic Waves in Multilayered Media", IEEE Trans UFFC, vol. 42, no. 4, pp. 525-542, July 1995.

[24] A. Neumaier, A., "Solving Ill-conditioned and Singular Linear Systems; A Tutorial on Regularization", IEEE Trans UFFC, vol. 44, no. 5, pp. 1018-1026, Sept 1998.

[25] G. Hayward and J.A. Hossack, "Unidimensional Modelling of 1-3 Composite Transducers", JASA, vol. 88, no. 2, pp. 599-607, 1990. 